\title{
Motion Control of a Four-wheel-drive Omnidirectional Wheelchair with High Step Climbing Capability
}

\author{
Masayoshi Wada \\ Dept. of Mechanical Systems Engineering \\ Tokyo University of Agriculture and Technology \\ Japan
}

\section{Introduction}

In recent years, aging problem has been arising to be among the most serious social issues world wide, especially in some European and Asian countries, involving Japan. It is reported in Japan that the population of over 65 years old would reach 30,000,000 in 2012 and grow over $30 \%$ of total population in 2025[1].

Electric wheelchairs, personal mobiles, scooters are currently commercially available not only for handicapped persons but also for elderly. However, such a rapid grow of aging populations suggest that requirements for electric mobile systems will soon increase dramatically for supporting mobility and activity of elderly people and reducing labor of care-givers.

However, those mobile systems do not have enough functionalities and capabilities for moving around existing environments including step, rough terrain, slopes, gaps, floor irregularities as well as insufficient traction powers and maneuverability in crowded areas. Promotion of barrier-free environments will be required for a large number of users of wheelchairs and other electric mobile systems however, re-constructing of the existing facilities could not be a feasible solution because of the limitations in economy and time.

For overcoming the problem, to improve the mobility of the electric mobile systems to adapt to existing environments could be one solution. For this objective, we propose a new type of wheelchair, four-wheel-drive (4WD) omnidirectional system, with enhanced step climb capability together with high maneuverability. In this chapter, omnidirectional control of a wheelchair with 4WD mechanism would be mainly discussed.

The mobile systems realizing holonomic and omnidirectional motion is one of the important research area in mobile robots. It provide flexibility and high maneuverability to motion planners and human drivers. The holonomic and omnidirectional mobile capability is very convenient for human drivers since they do not have to understand drive mechanisms and its configuration at all. A human only commands the direction and velocity of motion he/her wants to perform since a holonomic and omnidirectional mechanism can start to move in any direction with any configuration of the mechanism such as directions of wheels. 
This characteristics is vary suitable for wheelchairs and personal mobiles which is used for daily life for maneuvering crowded area at home.

In the following sections, a new type of omnidirectional system is proposed which realizes the holonomic and omnidirectional capability together with high mobility on irregular terrains or steps.

\section{Conventional Omnidirectional Systems For Wheelchairs}

A standard wheelchair cannot move sideways. It needs a complex series of movements resembling parallel automobile parking when a wheelchair user wants to move sideways. A lot of omnidirectional drive systems were developed and applied to electric wheelchairs to enhance standard wheelchair maneuverability by enabling them to move sideways without changing the chair orientation. In Fig. 1, an omnidirectional wheelchair with Mechanum wheels [2] uses barrel-shaped rollers on the large wheel's rim inclining the direction of passive rolling 45 degrees from the main wheel shaft and enabling the wheel to slide in the direction of rolling. The standard four-Mechanum-wheel configuration assumes a car-like layout. The inclination of rollers on the Mechanum wheel causes the contact point to vary relative to the main wheel, resulting in energy loss due to conflictions in motion among the four wheels. Because four-point contact is essential, a suspension mechanism is definitely needed to ensure 3-degrees-of-freedom (3DOF) movement. Fig.2 shows an omnidirectinal wheelchair with ball wheel mechanisms developed at MIT [3]. Each ball wheel is driven by an individual motor which provides active traction force in a specific direction while perpendicular to the active direction. With this drive system, the point of contact of a wheel is stable relative to the wheelchair body that enables accurate motion control and smooth movements with no vibration.

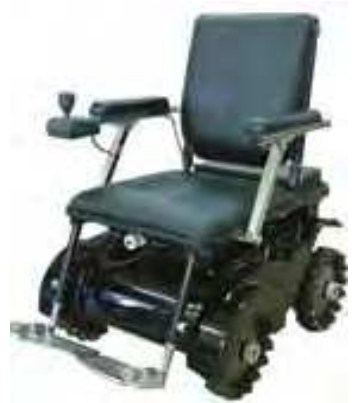

Fig. 1. Omnidirectional wheelchair with Mechanum wheels [2]

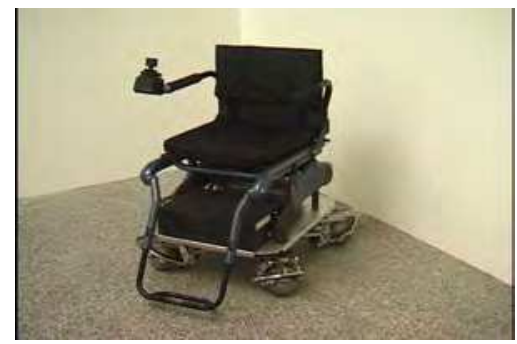

Fig. 2. Ball wheel omnidirectional wheelchair [3] 
The other omnidirectional mechanism is VUTON crawler[4] which consists of many cylindrical free rollers. Since VUTON mechanism allows the multiple rollers to touch the ground simultaneously, heavy load can be applied on the platform.

All of the above omnidirectional systems need one motor to drive one wheel mechanism therefore four motors are needed to drive a four-wheeled wheelchair, while a wheelchair has three degrees of freedoms (DOF) on the floor. Thus, it involves 1 DOF redundancy in actuation which causes conflictions in motion among the four wheels.

\section{Four-Wheel-Drive (4WD) Mechanism}

To give a high mobile capability to a wheelchair, we introduce a four-wheel-drive (4WD) mechanism to our omnidirectional mobile system. At first, the original 4WD design is simply mentioned.

The 4WD drive system was invented in 1989 [5] for enhancing the traction and step climbing capability of the differential drive systems which schematic is illustrated in Fig.3. This 4WD mechanism has recently applied to a product design by a Japanese company [6]. The wheelchair equips four wheels, two omni-wheels in front and two normal tires in rear. A normal wheel and an omni-wheel, mounted on the same side of the chair, are interconnected by a chain or a belt transmission to rotate in unison with a drive motor. A common motor is installed to drive normal and omni wheel pair via synchro-drive transmission on each side of the mechanism. Then two motors provide deferent velocity on each side witch presents differential drive motion of $4 \mathrm{WD}$ mechanism. Thus all four wheels on $4 \mathrm{WD}$ can provide traction forces. Since the center of rotation shifts backward, when it turns about a steady point on the floor, it requires large space when the wheelchair is controlled in the standard differential drive manner. The offset distance between drive wheels and a center of a chair makes the maneuverability of the wheelchair worse.

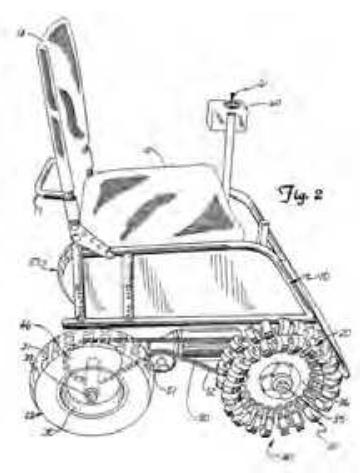

Fig. 3. Original 4WD synchronized transmission

\section{Powered-caster Omnidirectional Control}

We apply powered-caster control to $4 \mathrm{WD}$ mechanism to give an omnidirectional mobile capability to a wheelchair with $4 \mathrm{WD}$. In this section, The powered-caster omnidirectional control for the original single type configuration[7] is breafly mentioned followed by the control of 4WD mechanism in the next section. 


\subsection{Powered-caster Mechanism}

Fig.4 shows a top view of a powered-caster. The original design of the powered-caster is a single wheel type in which normal wheel is off-centered from steering shaft. The wheel shaft and the steering shaft of the powered-caster is driven by independent motors. When only the wheel shaft is rotated by the motor, the caster moves in forward direction which is denoted as $\dot{x}_{w}$ in Fig.4. When only the steering shaft is rotated by an another motor, the mechanism rotates about the point of contact which is also shown in the figure. By this motion of rotation, the steering shaft moves in lateral in $\dot{y}_{w}$ at the instant which is tangential of the circle which center is at the point of contact with the radius is s, the caster-offset.

These velocity vectors are independently controlled and directing right angle for each other. To generate a velocity $V$ in the direction $\theta$ at the center of the steering shaft, the wheel and the steering shaft rotations, $\omega_{\mathrm{w}}$ and $\omega_{\mathrm{s}}$, are derived by the following kinematics.

$$
\left[\begin{array}{l}
\omega_{w} \\
\omega_{s}
\end{array}\right]=\left[\begin{array}{ll}
\frac{1}{r} \cos \theta & -\frac{1}{r} \sin \theta \\
\frac{1}{s} \sin \theta & \frac{1}{s} \cos \theta
\end{array}\right]\left[\begin{array}{l}
\dot{x}_{w} \\
\dot{y}_{w}
\end{array}\right]
$$

where $s$ and $r$ are the caster offset and the wheel radius respectively. Thus shaft rotations are determined by a function of $\theta$, the relative angle between the desired direction and the wheel mechanism.

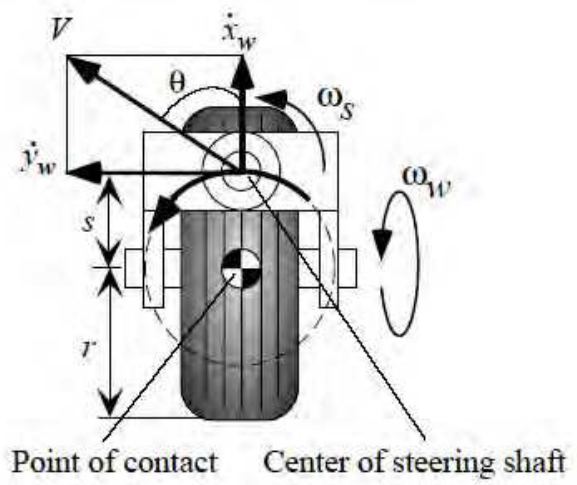

Fig. 4. Velocity control of a powered-caster

\subsection{Omnidirectional Mobile Robot with Powered-casters}

Figure 5 shows a schematic overview of an omnidirectional mobile robot with two poweredcasters. The robot with a pair of powered-casters is controlled by four electric motors which involves one redundant DOF in actuation. For this class of omnidirectional robots, the powered-caster provides an active traction force in an arbitrary direction for propelling the robot. To coordinate the multiple powered-casters, motors on a powered-caster are controlled based on the velocity based robot model. 
The inverse kinematics of two-wheeled mobile robot is represented as (2) which represents a relationship between the commanded robot velocity in $3 \mathrm{DOF}\left[\dot{x}_{v}, \dot{y}_{v}, \dot{\phi}_{v}\right]$ and a A wheel velocity $\left[\dot{x}_{a}, \dot{y}_{a}\right]$ and a B wheel velocity $\left[\dot{x}_{b}, \dot{y}_{b}\right]$.

$$
\left[\begin{array}{l}
\dot{x}_{a} \\
\dot{y}_{a} \\
\dot{x}_{b} \\
\dot{y}_{b}
\end{array}\right]=\left[\begin{array}{ccc}
1 & 0 & -\frac{W}{2} \cos \phi_{v} \\
0 & 1 & -\frac{W}{2} \sin \phi_{v} \\
1 & 0 & \frac{W}{2} \cos \phi_{v} \\
0 & 1 & \frac{W}{2} \sin \phi_{v}
\end{array}\right]\left[\begin{array}{l}
\dot{x}_{v} \\
\dot{y}_{v} \\
\dot{\phi}_{v}
\end{array}\right]
$$

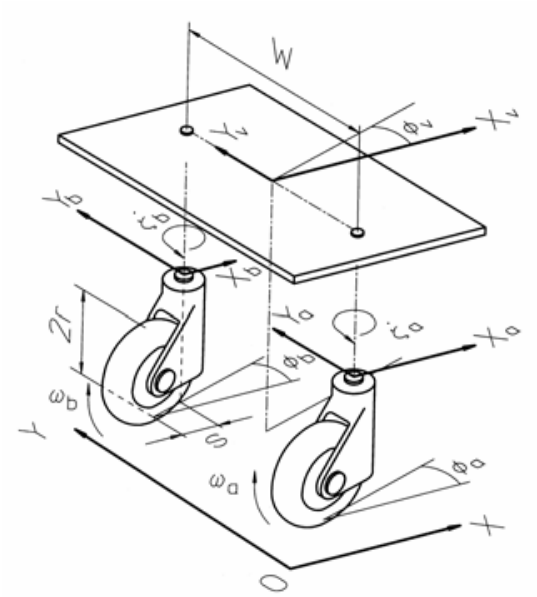

Fig. 5. A two-wheeled omnidirectional robot

\section{Omnidirectional Control of 4WD Mobile system}

In our project, it is a goal to develop an omnidirectional wheelchair with high mobility and maneuverability in a single design which can be used in multiple environments including outdoor and indoor. To enable a wheelchair to move in any direction instantaneously, omnidirectional control method, called "powered-caster control" which was introduced in previous section, is extended and applied to the 4WD mechanism [7]. Fig.6 shows a schematic of the 4WD omnidirectional wheelchair. The wheelchair has two omniwheels in front and standard pneumatic tires in rear which form $4 \mathrm{WD}$ configuration. A pair of an omniwheel and a pneumatic tire mounted on the same side of the wheelchair are connected by belt transmission for rotating unison and driven by a common motor which configuration is completely identical to the original 4WD system shown in Fig.3.

In our design, an additional third motor is mounted on the conventional 4WD platform for rotating a chair about the vertical axis which is also illustrated in Fig.6. Those three motors including two wheel motors and the chair rotation motor enable the wheelchair to realize independent 3DOF omnidirectional motion by a coordinated motion control [8],[9]. 
To achieve coordinated control for omnidirectional motion of a chair, the powered-caster omnidirectional control for a twin-caster configuration has been applied to the 4WD system. Fig.7 illustrates a schematic top view of a 4WD mechanism. In Fig.7, it is found that rear two drive wheels and center axis form a twin-caster configuration, i.e. parallel two wheels are located on the off-centered position which midpoint is distant from vertical steering axis, which is emphasized by thick lines in the Fig.7 and a vehicle with a twin caster drive mechanism is shown in Fig.8. The powered-caster omnidirectional control enables the caster mechanism to emulate the caster motion by actuating wheel and steering axes.

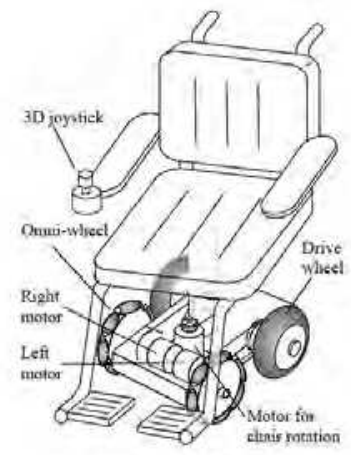

Fig. 6. A 4WD omnidirectional wheelchair

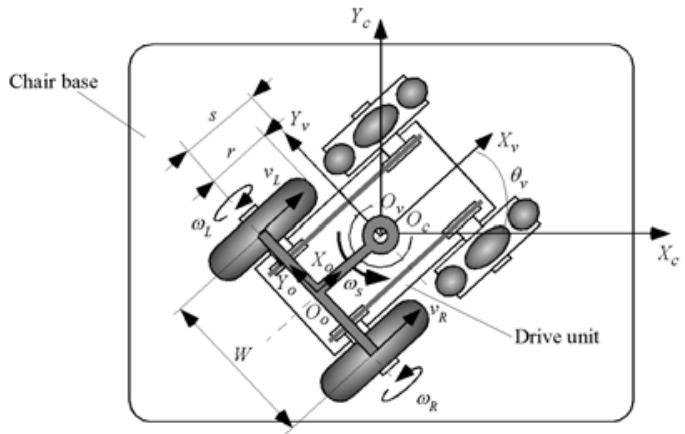

Fig. 7. Omnidirectional vehicle with 4WD mechanism

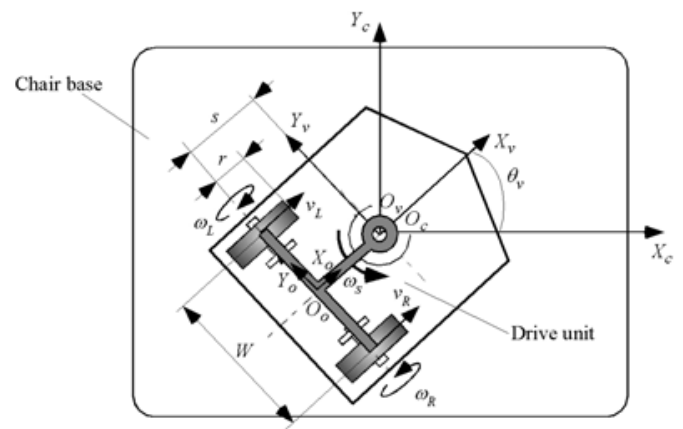

Fig. 8. Omnidirectional vehicle with a twin-caster 
The powered-caster-based coordinate control of three motors needs a kinematic model of the $4 \mathrm{WD}[10]$. The kinematic model represents the relationships between the motion of the $4 \mathrm{WD}$ and the three motor angular velocities of the drive wheels and the chair rotation axis. First, we consider the fundamental motions of a twin-caster drive (TCD). Figure 9a shows the translational motion of the vehicle in which two wheels rotate in same angular velocity to travel in same direction. In this case, TCD travels also straight forward, therefore TCD velocity and its rotation are represented as follows.

$$
\begin{aligned}
& \dot{x}_{v}=\frac{1}{2}\left(v_{R}+v_{L}\right)=v \\
& \dot{\phi}_{v}=\frac{1}{W}\left(v_{R}-v_{L}\right)=0
\end{aligned}
$$

Figure $9 \mathrm{~b}$ shows another motion in which two wheels are rotated at same angular velocities but in opposite directions resulting in spin of TCD about the midpoint of two wheels. This motion provides only rotation but no translation velocity which is represented as,

$$
\begin{aligned}
& \dot{x}_{v}=\frac{1}{2}\left(v_{R}+v_{L}\right)=0 \\
& \dot{\phi}_{v}=\frac{1}{W}\left(v_{R}-v_{L}\right)=\frac{2 v}{W}
\end{aligned}
$$

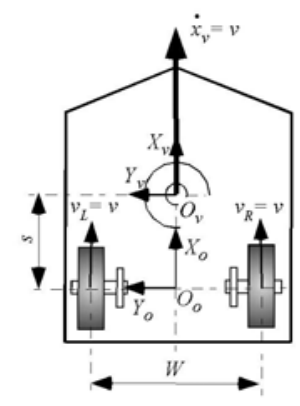

(a): Motion in X-direction

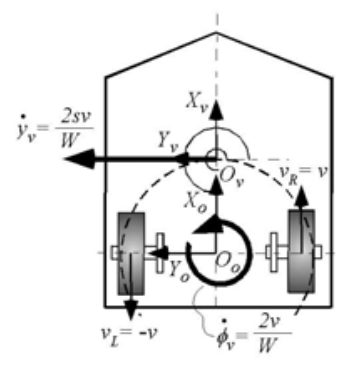

(b): Motion in Y-direction

Fig. 9. Omnidirectional control for twin-caster drive (TCD)

Now we focus on the motion of the steering center whose location is identical to the rotation center of a chair. When TCD rotates about the midpoint of two wheels, the center of the steering presents a circular motion whose center is at the midpoint and the radius equals the caster offset, s. At each moment, velocity at the center is directing tangential direction of the circle which directs the lateral direction of TCD at all times. The lateral velocity denoted by $\dot{y}_{v}$ in Fig. $9 \mathrm{~b}$ is represented by,

$$
\dot{y}_{v}=s \dot{\phi}_{v}=\frac{s}{W}\left(v_{R}-v_{L}\right)=\frac{2 s v}{W}
$$


The translation velocities $\dot{x}_{v}$ and $\dot{y}_{v}$ are directed at right angles to each other. Note here, the rotation of TCD is not independently controlled since the rotation $\dot{\phi}_{v}$ is determined by creating the lateral velocity $\dot{y}_{v}$ to satisfy Eq (5). From Eqs. (3)-(5), the relationships between the vehicle translation velocity and wheel velocities are derived as,

$$
\left[\begin{array}{l}
\dot{x}_{v} \\
\dot{y}_{v}
\end{array}\right]=\left[\begin{array}{cc}
1 / 2 & 1 / 2 \\
s / W & -s / W
\end{array}\right]\left[\begin{array}{l}
v_{R} \\
v_{L}
\end{array}\right]
$$

Thus, translation velocities along the $\mathrm{X}$ - and $\mathrm{Y}$-directions are completely determined and independently controlled by wheel velocities. To generate the required velocity vector that directs in an arbitrary direction with arbitrary magnitude, the reference vector is projected in X- and Y-directions of vehicle coordinate system (Fig.10). The velocity component in each direction, $\dot{x}_{v}$ or $\dot{y}_{v}$, can be independently achieved by using kinematics of TCD in Eq. (6).

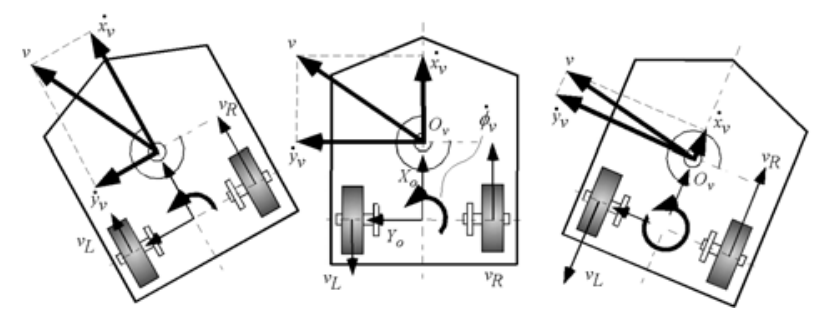

Fig. 10. Projection of a command velocity into vehicle coordinate system depending on the TCD orientation.

When the reference velocity is steady to the ground, the velocity components in X- and Ydirections vary depending on the TCD orientation relative to the ground. Therefore, wheel velocities also vary which results in straight motion of the TCD center (see Fig. 11). TCD shows spontaneous flipping behavior during the motion, which is often found in passive casters installed on legs of chairs and tables, etc. It is said the powered-caster control emulates caster motion by actively actuating the wheel axis.

Fig.11 shows a one of the simulation results in which an omnidirectional control of the twincaster mechanism are tested. In the simulation, twin-caster mechanism is controlled to track a straight line with a center of a mechanism locating on the line at all times. During the motion, the orientation of the mechanism is rapidly flipped over and orient to the direction of motion. This flip motion is often seen on passive casters which installed on the legs of office chairs and tables. Thus the powered-caster control achieves the emulation of caster motions by coordinated control of multiple actuators. 


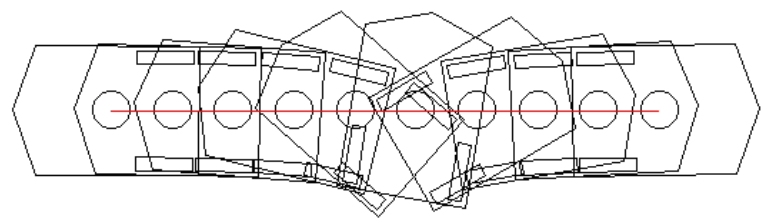

Fig. 11. Omnidirectional control for twin-caster mechanism

Translation in arbitrary direction is achieved by TCD as presented above. However, orientation of TCD can not be controlled independently by the wheel rotations. To control 3DOF motion of a chair, the chair rotation axis must be also coordinated. The velocity command is given based on the chair orientation since a joystick is fixed on the chair. Then the command velocity is translated into TCD coordinate by the relative orientation of the chair to the vehicle, $\theta_{\mathrm{v}}$. as

$$
\begin{aligned}
& \dot{x}_{c}=\dot{x}_{v} \cos \theta_{v}-\dot{y}_{v} \sin \theta_{v} \\
& \dot{y}_{c}=\dot{x}_{v} \sin \theta_{v}+\dot{y}_{v} \cos \theta_{v} \\
& \dot{\theta}_{c}=\dot{\theta}_{v}+\omega_{s}
\end{aligned}
$$

From eqs. (6) and (7), an overall kinematic model of the omnidirectional 4WD wheelchair is represented as follows.

where,

$$
\left(\begin{array}{l}
\dot{x}_{c} \\
\dot{y}_{c} \\
\dot{\theta}_{c}
\end{array}\right)=\left(\begin{array}{ccc}
J_{11} & J_{12} & 0 \\
J_{21} & J_{22} & 0 \\
r / W & -r / W & 1
\end{array}\right)\left(\begin{array}{c}
\omega_{R} \\
\omega_{L} \\
\omega_{S}
\end{array}\right)
$$

$$
\begin{aligned}
& J_{11}=\frac{r \cos \theta_{v}}{2}-\frac{r s \sin \theta_{v}}{W} \\
& J_{12}=\frac{r \cos \theta_{v}}{2}+\frac{r s \sin \theta_{v}}{W} \\
& J_{21}=\frac{r \sin \theta_{v}}{2}+\frac{r s \cos \theta_{v}}{W} \\
& J_{22}=\frac{r \sin \theta_{v}}{2}-\frac{r s \cos \theta_{v}}{W}
\end{aligned}
$$

Where $r, W$ and $s$ are the wheel radius, tread and caster-offset, respectively. A $3 \times 3$ matrix in the right side of eq.(8), called as Jacobian, is a function of orientation of the 4WD unit with relative to the chair base, $\theta_{\mathrm{v}}$. All elements in the Jacobian can always be calculated and a determinant of the Jacobian may not be zero for any $\theta_{\mathrm{v}}$. Therefore there is no singular point on the mechanism and an inverse Jacobian always exits. The three motors are controlled to realize a 3DOF angular velocity commands $\dot{x}_{v}, \dot{y}_{v}$ and $\dot{\phi}_{v}$ by independent speed controllers 
for omnidirectional movements. Thus, holonomic 3DOF motion can be realized by the proposed mechanism. This class of omnidirectional mobility, so called "holonomic mobility", is very effective to realize the high maneuverability of wheelchairs by an easy and simple operation.

\section{Prototype Design}

\subsection{Mechanical Design}

Wheelchair specifications for prototype design are shown in Table 1. The wheelbase and tread of the $4 \mathrm{WD}$ mechanism are $400 \mathrm{~mm}$ and $535 \mathrm{~mm}$ respectively. Those dimensions are determined to satisfy the limitation of the standard wheelchair specification for the dimension, $600 \mathrm{~mm}$ in width and $700 \mathrm{~mm}$ in length as shown in the spec. The required step height which can be surmounted by the wheelchair is approx. $100 \mathrm{~mm}$ for accessing to a train car from a station platform with no assistance. The maximum running speed for continuous drive is $6 \mathrm{~km} / \mathrm{h}$ which is same as conventional standard wheelchairs in Japan.

Fig.12 illustrates a 3D drawing of a prototype designed by 3D CAD. Fig.13 shows an overview of the prototype wheelchair.

\begin{tabular}{|l|l|l|}
\hline \multirow{3}{*}{ Dimension } & Width & $600 \mathrm{~mm}$ \\
\cline { 2 - 3 } & Length & $700 \mathrm{~mm}$ \\
\cline { 2 - 3 } & Height & $450 \mathrm{~mm}$ \\
\hline \multirow{2}{*}{ Weight } & Total & $180 \mathrm{~kg}$ (human+wheelchair) \\
\cline { 2 - 3 } & Wheelchair & $100 \mathrm{~kg}$ (including batteries) \\
\hline Speed & $6 \mathrm{~km} / \mathrm{h}$ (Max.) \\
\hline Surmountable step & $100 \mathrm{~mm}$ in height \\
\hline
\end{tabular}

Table 1. Wheelchair Specifications

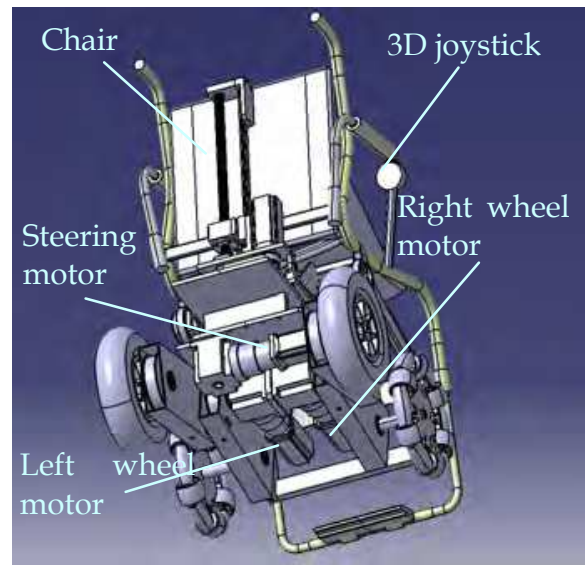

Fig. 12. Prototype bottom view by 3D CAD 


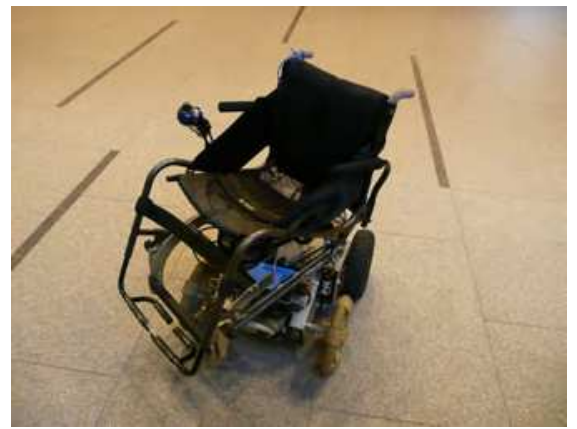

Fig. 13. 4WD omnidirectional wheelchair prototype

\subsection{Control system}

Figure 14 shows a control system of the wheelchair prototype. Most of equipments including a controller, motors, motor drivers, a battery and sensors are installed on 4WD mechanism side. Electric power is provided by a car battery and distributed to all components on the chair after the inversion to AC 100V. A tablet PC controls three motors to realize an omnidirectional and holonomic motions of a wheelchair based on reference velocity commanded by a $3 \mathrm{D}$ joystick. A velocity command is sent to each motor driver via a D/A interface while a encoder pulse is sent back to the PC via a pulse counter interface which form a velocity feedback loop of the axis. An absolute encoder is installed only on the chair rotation shaft which detects relative angle between the 4WD and the chair which needs no initialization process at power on reset. Since a chair have to rotate continuously with no mechanical limit, slip rings are also installed on the chair rotation axis. A USB hub on the chair side enables extension of devices additional to an A/D converter for the 3D joystick.

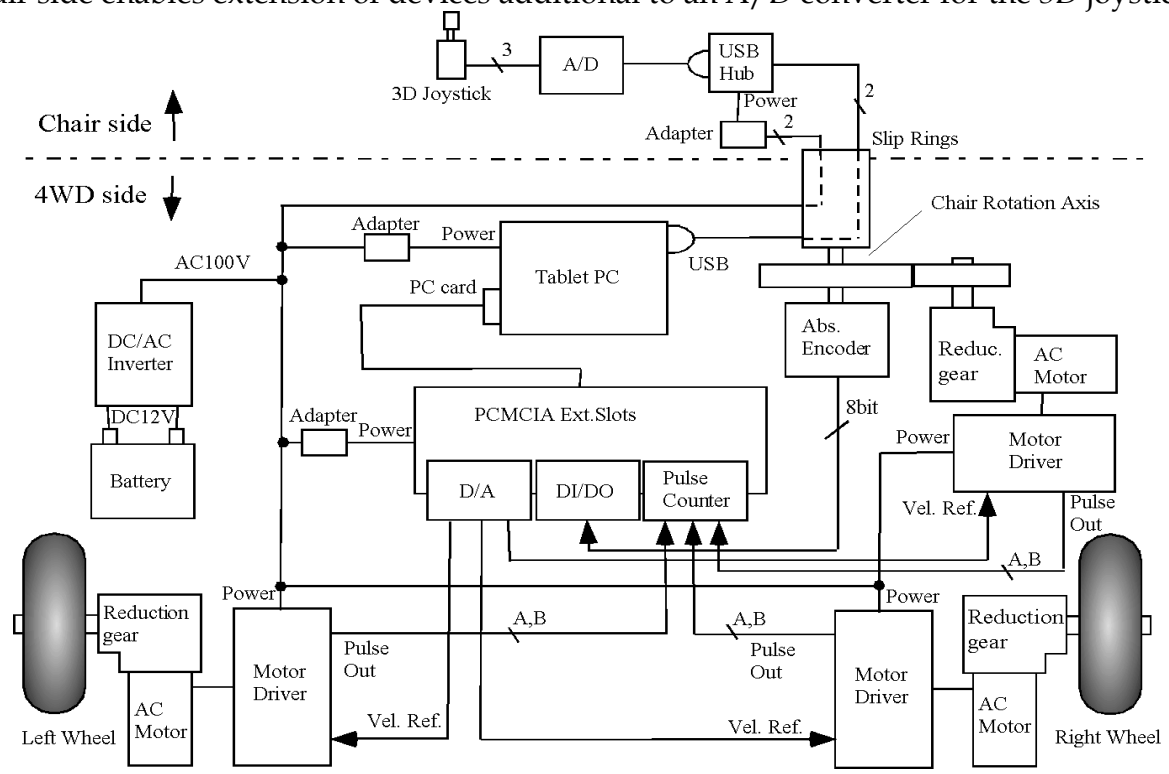

Fig. 14. System configuration of the prototype wheelchair 


\section{Experiments}

\subsection{Omnidirectional motion}

To verify the omnidirectional mobility of the proposed system, lateral motion was presented by the prototype wheelchair while keeping the chair orientation constant.

A small ball was attached to the chair to measure the location of the center of the steering axis. A stereo camera system (Quick Mag IV from OKK Inc, Japan) detects the ball location in 3D coordinates. The prototype was controlled by a remote PC via a LAN connection through which the remote PC send the velocity command to the wheelchair controller. Thus, a complete linear trajectory in lateral direction was commanded to the prototype. Figure 15 shows a chair motion detected by the stereo camera system. An actual and a reference path are plotted in the figure which closely agree. Figure 16 shows tracking errors between the actual and the reference. The error was suppressed to within $10 \mathrm{~mm}(+5$ to $-5 \mathrm{~mm})$ despite the flipping behavior of the $4 \mathrm{WD}$ occurred during the experiment motion.

The camera system also provided real-time video images with over-writing rectangle window(s) and the path of the target(s) (in this experiment, a small ball). Figure 17(a)-(f) shows a series of video images. The prototype wheelchair moved from the right side of the picture frame to the left. The final picture, Fig. 17(f) shows a straight path which was created by the wheelchair movement. During the motion, the flipping behavior of the $4 \mathrm{WD}$ is found in Fig. 17(b), (c) and (d), which is also shown in a computer simulation in Fig. 11.

\subsection{Variable center of rotation}

The proposed system realizes holonomic and omnidirectional motion of a chair by the coordinated control of three motors. The holonomic mobile capability makes it possible to change the location of a center of rotation at any point to fit to customer requests. In a normal setup, a chair rotates about its center when the operator commands a spin turn by twisting the joystick. However, the location of the center is variable in the control program to any point including the out-of-footprint area of the wheelchair. However, usual requests may be to shift it to the back side to simulate a rear drive wheelchair, or to the front side to simulate a front drive wheelchair. To present the flexibility of the proposed system, two patterns of spin turn motion were performed. Figure 18(a) and (b) shows the final image of each test run provided by the stereo camera system. In Fig. 18(a), the wheelchair rotated about the front position which was located approx. $500 \mathrm{~mm}$ forward of the center of the chair. In Fig. 18(b), the center was shifted also approx. $500 \mathrm{~mm}$ towards the rear. From these results, the center of rotation can be customized to an individual.

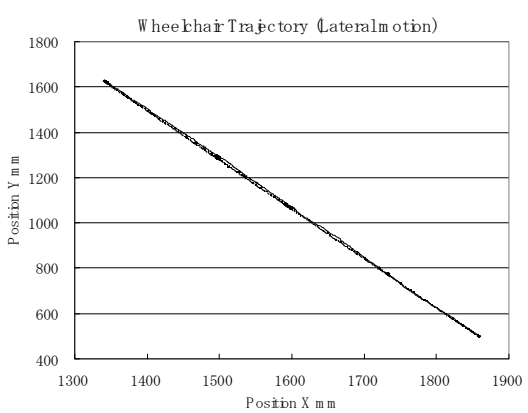

Fig. 15. Reference and actual trajectories of the 4WD wheelchair 


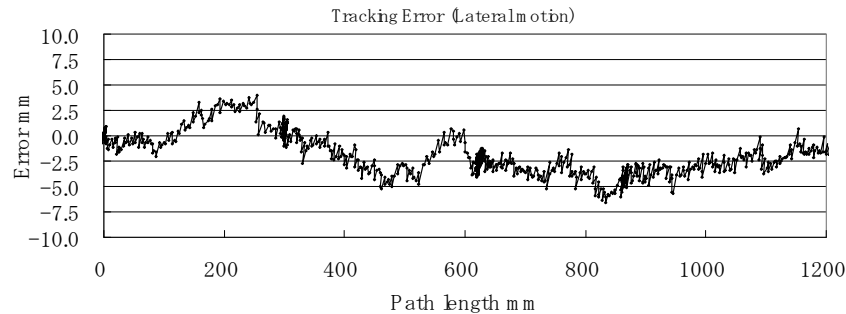

Fig. 16. Tracking error on the experiment using 4WD wheelchair

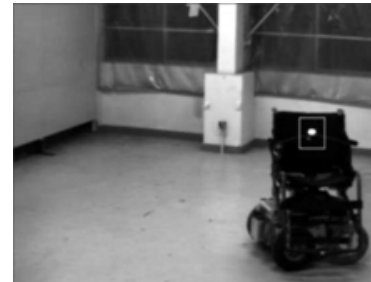

(a)

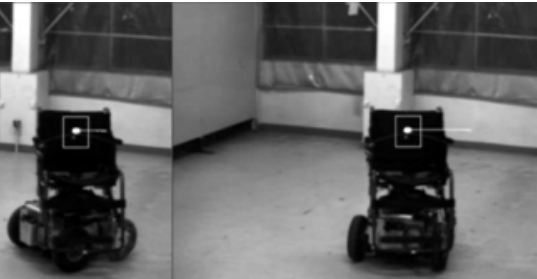

(c)

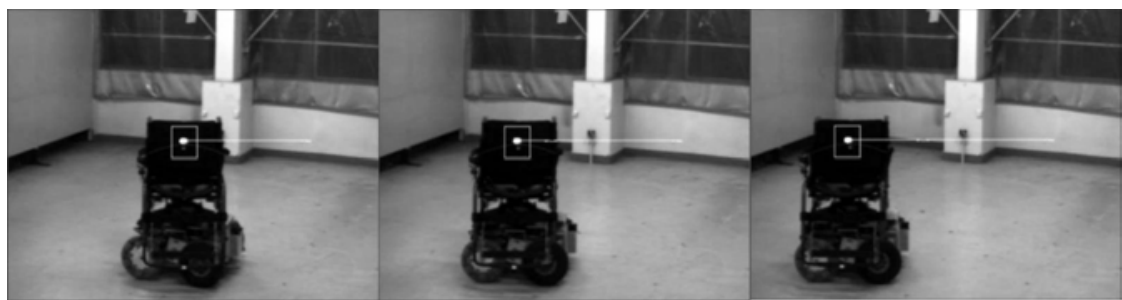

(d)

(e)

(f)

Fig. 17. An example of omnidirectional motion 1: the lateral motion of the wheelchair prototype; it moves in sideways from the right side to the left of the picture frames while maintaining the chair orientation to be stable.

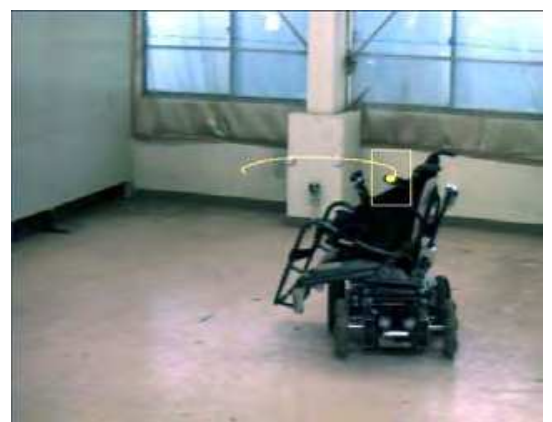

(a) Spin turn about the front position

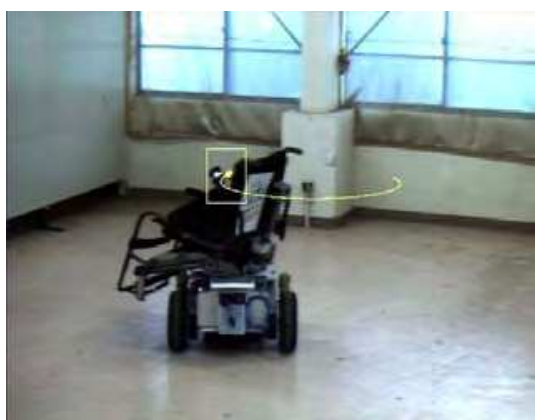

(b) Spin turn about the back position

Fig. 18 Variable center of rotation 


\subsection{Task example}

The holonomic and omnidirectional mobile systems are easy to maneuver because 3D command in X- and Y-directions and rotation are directory commanded and an operator does not have to consider the wheel motions and its configurations.

To demonstrate maneuverability, a task example was performed by the prototype. The task of getting out of a room by pulling the door open is one of difficult tasks for a wheelchair user. A task of pulling the door is relatively more difficult than pushing the door. This task example includes sub tasks such as: 1) approaching to the door to grasp the door knob, 2) moving backward to pull the door open, 3) going through the door and getting out of the room, and 4) driving to another location.

Figure 19(a)-(j) show screen shots of the experiment. In this test, the prototype wheelchair was operated using a 3D joystick by a human operator who was sitting on the chair. Since the wheelchair has high maneuverability, the task was successfully achieved with no collision with the door or a wall. The operator did not see the 4WD mechanism during the task, however, the 4WD mechanism changed its orientation widely when the operator moved sideways to avoid colliding with the door (Fig. 19(d)-(g)).

Figure 20 shows the 3D commands (X, Y and Rotation) to the wheelchair during the task of Fig.19 which shows 3D simultaneous motion to complete the door opening task. Those are found in sub tasks including, 1) approaching the door and 4-1) turning after exiting the room. Individual lateral translations are often found in subtask 3) exiting the room.

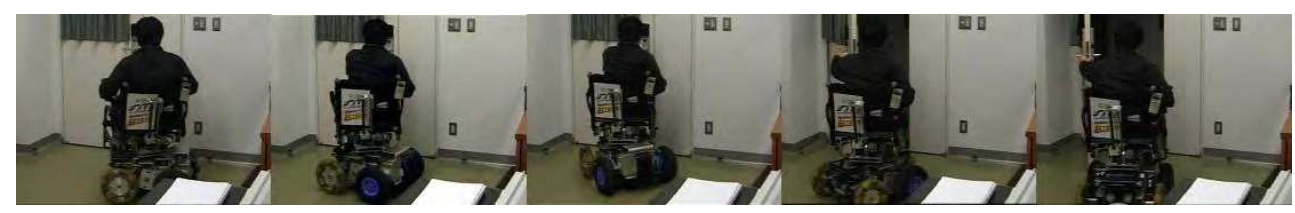

(a)

(b)

(c)

(d)

(e)

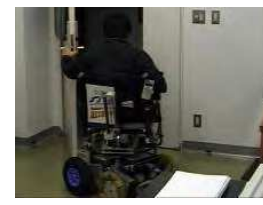

(f)

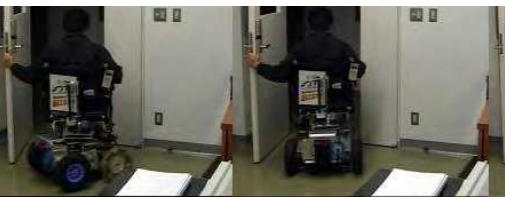

(g) (h)

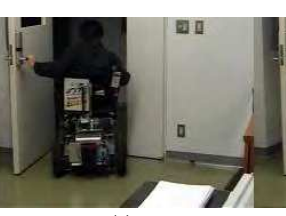

(i)

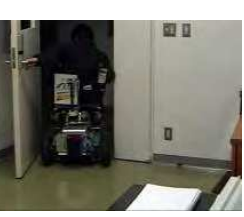

(j)

Fig. 19. An example of tasks using a electric wheelchair: Getting out of a room with pulling a door open. 


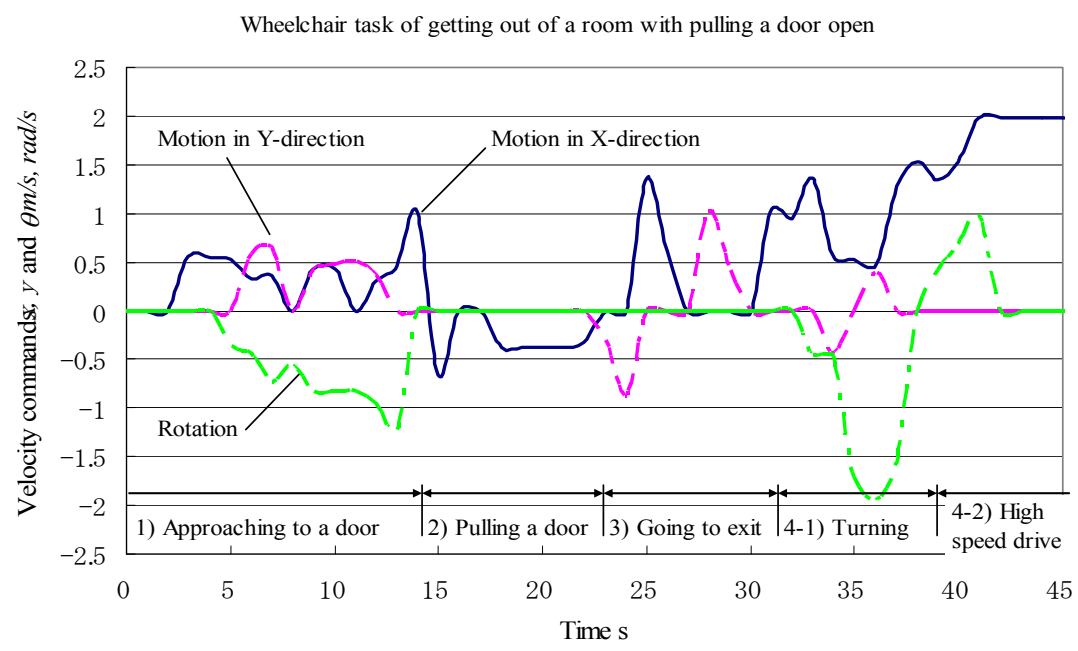

Fig. 20. 3DMotion of wheelchair presenting a door opening task for exiting a room

\section{Conclusion}

Mechanism and omnidirectional control of a 4WD mechanism for wheelchairs are presented in this chapter. The omnidirectional wheelchair system is proposed for improving maneuverability of standard wheelchairs The 4WD mechanism has high mobility which equips four wheels, two omni-wheels in the front and two normal tires in the rear, and all wheels provide traction even with two motors to drive these wheels. To realize holonomic and omnidirectional motion of a chair by utilizing the 4WD mechanism, the proposed system includes the third motor to rotate the chair at the center of the $4 \mathrm{WD}$ mechanism about the vertical axis.

For omnidirectional control of the $4 \mathrm{WD}$ mechanism, powered-caster control has been applied. To achieve a coordinated control of three motors, kinematics of the 4WD wheelchair was analyzed and a kinematic model was derived which represents the relationships between 3DOF wheelchair motion and the rotations of three motors. In the powered-caster control, two wheel motors are coordinated to translate the center of the chair in an arbitrary direction while the chair orientation is controlled by the third motor separately.

The omnidirectional motion was verified by a series of experiments using a wheelchair prototype. First, omnidirectional mobility was tested in which the wheelchair made a lateral motion without changing its orientation. Next, one of a applications of the holonomic mobility was performed in which the center of rotation was varied by a control program to customize per user request for simulating the wheelchair drive types, such as front drive, rear drive, or center drive.

To present the high maneuverability of the proposed omnidirectional mobile system, a task example was performed in which an operator maneuvered the wheelchair by 3D joystick to exit a room with pulling the door open. The task was successfully achieved with no collision 
with the door or walls. During the task, it was found that the simultaneous 3DOF motions, lateral translation are often commanded as well as the forward translation for pursuing the task.

From these experiments, the omnidirectional and holonomic mobile capability are shown to be very effective and useful for maneuvering in crowded areas and achieving complicated tasks.

\section{Acknowledgment}

This project was supported by the Industrial Technology Research Grant Program in 2006 from the New Energy and Industrial Technology Development Organization (NEDO), Japan, research ID 05A06715a.

\section{References}

[1] Population Statistics Japan 2006, by the National Institute of Population and Social Security Research, Japan.

[2] All-direction Power-driven Chair "FJ-UEC-600", Fujian Fortune Jet Mechanical \& Electrical Technology Co., Ltd.

[3] M. Wada and H. H. Asada (1999) Design and Control of a Variable Footprint Mechanism for Holonomic and Omnidirectional Vehicles and its Application to Wheelchairs, IEEE Trans on Robotics and Automation, Vol.15, No. 6, pp. 978-989.

[4] S. Hirose and S. Amano (1993) The VUTON : High Payload High Efficiency Holonomic Omni-Directional Vehicle, 6th Int. Symp. on Robotics Research.

[5] Jefferey Farnam (1989), Four-wheel Drive Wheel-chair with Compound Wheels, US patent $4,823,900$.

[6] Kanto Automobile Corp. "Patrafour" http://www.kanto-aw.co.jp/jp/products/wheelchair/

[7] M. Wada and S. Mori (1996), Holonomic and Omnidirectional Vehicle with Conventional Tires, Proceedings of the 1996 IEEE International Conference on Robotics and Automation (ICRA96), pp. 3671-3676.

[8] M. Wada, A. Takagi and S. Mori (2000), Caster Drive Mechanisms for Holonomic and Omnidirectional Mobile Platforms with no Over Constraint, Proceedings of the 2000 IEEE International Conference on Robotics and Automation (ICRA2000), pp. $1531-1538$.

[9] M. Wada (2007), Omnidirectional and Holonomic Mobile Platform with Four-WheelDrive Mechanism for Wheelchairs, JSME Journal of Robotics and Mechatronics, Vol. 19, No. 3, pp. 264-271.

[10] M. Wada (2007), Holonomic and Omnidirectional Wheelchairs with synchronized 4WD Mechanism, Proceedings of the 2007 IEEE/RSJ International Conference on Intelligent Robots and Systems (IROS2007), pp. 1196-1202. 


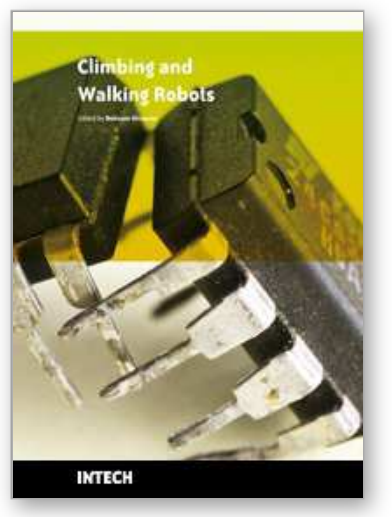

\author{
Climbing and Walking Robots \\ Edited by Behnam Miripour
}

ISBN 978-953-307-030-8

Hard cover, 508 pages

Publisher InTech

Published online 01, March, 2010

Published in print edition March, 2010

Nowadays robotics is one of the most dynamic fields of scientific researches. The shift of robotics researches from manufacturing to services applications is clear. During the last decades interest in studying climbing and walking robots has been increased. This increasing interest has been in many areas that most important ones of them are: mechanics, electronics, medical engineering, cybernetics, controls, and computers. Today's climbing and walking robots are a combination of manipulative, perceptive, communicative, and cognitive abilities and they are capable of performing many tasks in industrial and non- industrial environments.

Surveillance, planetary exploration, emergence rescue operations, reconnaissance, petrochemical applications, construction, entertainment, personal services, intervention in severe environments, transportation, medical and etc are some applications from a very diverse application fields of climbing and walking robots. By great progress in this area of robotics it is anticipated that next generation climbing and walking robots will enhance lives and will change the way the human works, thinks and makes decisions. This book presents the state of the art achievments, recent developments, applications and future challenges of climbing and walking robots. These are presented in 24 chapters by authors throughtot the world The book serves as a reference especially for the researchers who are interested in mobile robots. It also is useful for industrial engineers and graduate students in advanced study.

\title{
How to reference
}

In order to correctly reference this scholarly work, feel free to copy and paste the following:

Masayoshi Wada (2010). Motion Control of a Four-wheel-drive Omnidirectional Wheelchair with High Step Climbing Capability, Climbing and Walking Robots, Behnam Miripour (Ed.), ISBN: 978-953-307-030-8, InTech, Available from: http://www.intechopen.com/books/climbing-and-walking-robots/motion-control-of-a-four-wheeldrive-omnidirectional-wheelchair-with-high-step-climbing-capability

\section{INTECH}

open science | open minds

\section{InTech Europe}

University Campus STeP Ri

Slavka Krautzeka 83/A

51000 Rijeka, Croatia

Phone: +385 (51) 770447

Fax: +385 (51) 686166

\section{InTech China}

Unit 405, Office Block, Hotel Equatorial Shanghai

No.65, Yan An Road (West), Shanghai, 200040, China 中国上海市延安西路65号上海国际贵都大饭店办公楼 405 单元

Phone: +86-21-62489820

Fax: $+86-21-62489821$ 
www.intechopen.com 
(C) 2010 The Author(s). Licensee IntechOpen. This chapter is distributed under the terms of the Creative Commons Attribution-NonCommercialShareAlike-3.0 License, which permits use, distribution and reproduction for non-commercial purposes, provided the original is properly cited and derivative works building on this content are distributed under the same license. 